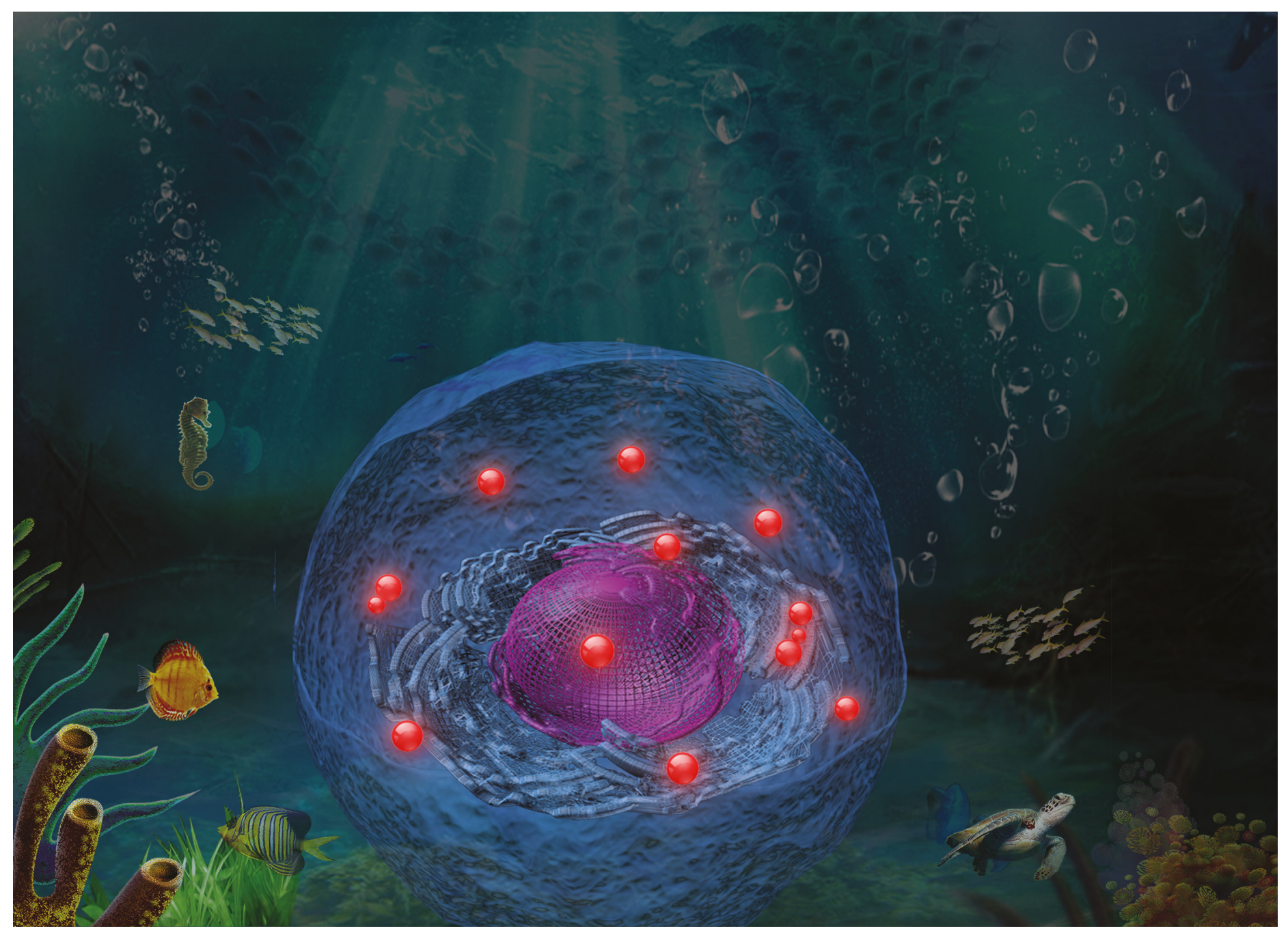

Showcasing research from Dr Sijie Chen et al., Ming Wai Lau Centre for Reparative Medicine, Karolinska Institutet, Hong Kong

A near-infrared AIE probe for super-resolution imaging and nuclear lipid droplet dynamic study

A near-infrared AIE probe was developed for super-resolution imaging of lipid droplets (LDs) and dynamic study of nuclear LDs (nLDs) in physiological process. This work provides a promising tool for LDs studies and contributes to a better understanding of nLDs.

\section{As featured in:}

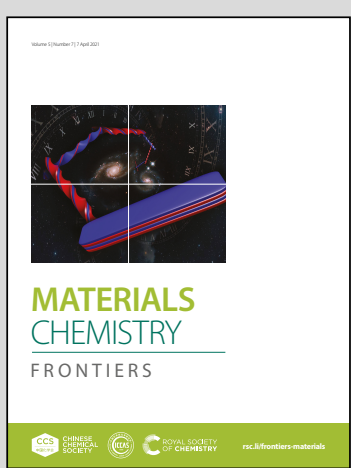

See Sijie Chen et al.,

Mater. Chem. Front., 2021, 5, 3043. 
Check for updates

Cite this: Mater. Chem. Front., $2021,5,3043$

Received 8th November 2020, Accepted 10th February 2021

DOI: $10.1039 / \mathrm{d} 0 \mathrm{qm} 00914 \mathrm{~h}$

rsc.li/frontiers-materials

\title{
A near-infrared AIE probe for super-resolution imaging and nuclear lipid droplet dynamic study $\dagger$
}

\author{
Ming-Yu Wu, (D) $\ddagger^{\mathrm{ab}}$ Jong-Kai Leung, (D) $\ddagger^{\mathrm{b}}$ Chuen Kam, (D) ${ }^{\mathrm{b}}$ Tsu Yu Chou, (D) ${ }^{\mathrm{b}}$ \\ Dong Wang, (D) ${ }^{c}$ Shun Feng ${ }^{a}$ and Sijie Chen (iD *b
}

\begin{abstract}
In contrast to the lipid droplets (LDs) in the cytoplasm, nuclear lipid droplets (nLDs), generated from the metabolically active inner nuclear membrane, contribute to the nuclear lipid homeostasis, locally regulate the availability of signaling lipids, and exchange the proteins between LDs and nuclei. Since the currently available fluorescence tools for imaging and tracking nLDs are limited, exploring a new nLD probe would be highly beneficial for studying the biogenesis and functional significance of nLDs. Herein, we developed a near-infrared probe, namely DTZ-TPA-DCN, which possessed the aggregation-induced emission (AIE) property with good biocompatibility for cytoplasm LD and nLD imaging. DTZ-TPA-DCN could be used for the super-resolution imaging of LDs with the structured illumination microscopy. By labelling nLDs, we could monitor the nLD formation under an ER stress, and for the first time, we observed that the signaling lipid, namely diacylglycerol (DAG), enhanced the formation of the nLDs. Together, DTZ-TPA-DCN provided a promising imaging tool for nLD studies, which contributed to a better understanding of the physiological significance of nLDs.
\end{abstract}

\section{Introduction}

Lipid droplets (LDs) are indispensable in lipid metabolism and energy storage. ${ }^{1}$ Although they are primarily responsible for the lipid storage, LDs are also crucial in numerous biological processes, including the regulation of the protein degradation, ${ }^{2}$ the homeostasis of the plasma membrane, ${ }^{3}$ and cell signaling. ${ }^{4}$ In addition, LDs play a protective role against lipotoxicity and endoplasmic reticulum (ER) stress. ${ }^{5}$ Moreover, researches have also revealed that LDs are implicated in the progression of numerous diseases, such as cancer, ${ }^{6}$ diabetes, ${ }^{7}$ fatty liver disease, ${ }^{8}$ atherosclerosis, ${ }^{9}$ hyperlipidemia, ${ }^{10}$ and neurodegeneration. ${ }^{11}$

In general, LDs are thought to be originated from ER and further translocated to cytoplasm. ${ }^{12}$ However, a sub-population of LDs are found inside the nucleus of hepatocytes. ${ }^{13}$ These nuclear lipid droplets (nLDs) are derived from apolipoprotein B-free lumenal LDs, which highly accumulate inside the inner nuclear membrane (INM) upon an ER stress. ${ }^{14}$ In yeasts, on the

\footnotetext{
${ }^{a}$ School of Life Science and Engineering, Southwest Jiaotong University, Chengdu 610031, China

${ }^{b}$ Ming Wai Lau Centre for Reparative Medicine, Karolinska Institutet, Hong Kong. E-mail: sijie.chen@ki.se

'Center for AIE Research, College of Materials Science and Engineering, Shenzhen University, Shenzhen 518060, China

$\dagger$ Electronic supplementary information (ESI) available: Synthesis processes and characterization, photophysical properties, cell viability, and cell imaging results. See DOI: $10.1039 /$ d0qm00914h

\$ These authors contributed equally to this work.
}

other hand, nLDs are generated in the metabolically active territory of INM, which contributes to the nuclear lipid homeostasis. ${ }^{15}$ However, due to the limitation of research tools, the origin and physiological significance of nLDs are still poorly understood.

Fluorescence imaging has been widely used for visualizing biological molecules and structures, tracking physiological and pathological processes, and diagnosing diseases in biological systems. ${ }^{16}$ Conventional techniques, such as immunofluorescence and ectopically expressed genes fused with fluorescent protein, are commonly used to monitor the LDs in nucleus, which involve time-consuming experimental procedures. ${ }^{17}$ Although a considerable number of fluorescent probes have been developed recently for imaging cytoplasmic LDs, BODIPY 493/503 is the only one used for imaging nLDs. ${ }^{18}$ Nevertheless, most of the conventional dyes suffer from the inherent aggregation-caused quenching (ACQ) property of being easily self-quenched when the luminophores are aggregated. Probes with aggregation-induced emission (AIE) properties have received wide attention since the first reporting of the phenomenon in 2001. ${ }^{19}$ Compared with the ACQ fluorophores, AIE luminophores show weak or undetectable emissions in dilute solutions but emit strong fluorescence in the aggregated or solid states. These characteristics of AIE luminophores allow the fluorescence imaging with low background, high signal-to-noise ratio, and good photostability, making them ideal tools for the fluorescence imaging of biological targets and even the ultrastructural characterization of biological targets via superresolution imaging. ${ }^{20}$ 
In this contribution, a near-infrared (NIR) fluorescent probe, namely DTZ-TPA-DCN, for nLD imaging was developed based on a $\mathrm{D}-\pi-\mathrm{A}$ framework, in which non-planar triphenylamine (TPA) was the donor to evade the ACQ effect and the lipophilic cyano groups were the acceptors. Furthermore, the bithiophene and carbon-carbon double bonds were conjugation groups that could extend the absorption or emission wavelength. ${ }^{19 e}$

DTZ-TPA-DCN exhibited AIE properties and could be used for imaging LDs in both live and fixed cells with high selectivity. By using DTZ-TPA-DCN, we could clearly observe the LDs in the nucleus of cells in two-dimensional (2D) and three-dimensional (3D) images. Thanks to its good photostability, DTZ-TPA-DCN could also be used for structured-illumination microscopy (SIM)based super-resolution imaging of LDs. Furthermore, the dynamics of LDs inside the nucleus was also highlighted and investigated using DTZ-TPA-DCN.

\section{Results and discussion}

\section{Synthesis and photophysical properties}

DTZ-TPA-DCN was synthesized via a simple Knoevenagel condensation reaction of aldehyde and malononitrile (Scheme 1) with $87.5 \%$ yield. It was characterized by ${ }^{1} \mathrm{H}$ NMR, ${ }^{13} \mathrm{C}$ NMR and high-resolution mass spectrometry (ESI $\dagger$ ). The optical properties of DTZ-TPA-DCN are illustrated in Fig. 1. From photoluminescence spectrometry results, it was evident that DTZ-TPADCN showed its maximum absorption peak at $495 \mathrm{~nm}$ and near-infrared emission peak at $715 \mathrm{~nm}$ with a large Stokes shift of $220 \mathrm{~nm}$ (Fig. 1A). The emission spectra displayed a strong solvent effect from toluene $(605 \mathrm{~nm})$ to acetonitrile $(700 \mathrm{~nm})$, representing a $105 \mathrm{~nm}$ bathochromic shift (Fig. S1, ESI $\dagger$ ). To further investigate the AIE features of DTZ-TPA-DCN, acetonitrile/ water $\left(\mathrm{MeCN} / \mathrm{H}_{2} \mathrm{O}\right)$ solvent mixture with different $\mathrm{H}_{2} \mathrm{O}$ fractions was utilized as the solvent system. As depicted in Fig. 1B and C, DTZ-TPA-DCN showed a weak fluorescence emission at $700 \mathrm{~nm}$ with a $0.04 \%$ quantum yield (QY) in a pure MeCN solution. When adding $\mathrm{H}_{2} \mathrm{O}$ to the MeCN solution, the maximum emission gradually red-shifted from $700 \mathrm{~nm}$ to $715 \mathrm{~nm}$, and the fluorescence intensity slowly decreased at first as the water fractions were increased from $10 \%$ to $40 \%$. This result could be attributed to the twisted intramolecular charge transfer (TICT) effect. When the ratio of water in the mixed solvent was further increased from $40 \%$ to $90 \%$, the fluorescence intensity of DTZ-TPA-DCN rebounded dramatically due to the formation of the probe aggregates, and the fluorescence intensity of DTZ-TPA-DCN in the $90 \% \mathrm{H}_{2} \mathrm{O}$ solution (QY: $0.28 \%$ ) was 8.1-fold of that observed in pure MeCN solution. The aggregate formed with an average size of approximately $113.5 \mathrm{~nm}$ in diameter was confirmed via the dynamic light scattering technique (Fig. 1D). QY in the solid state

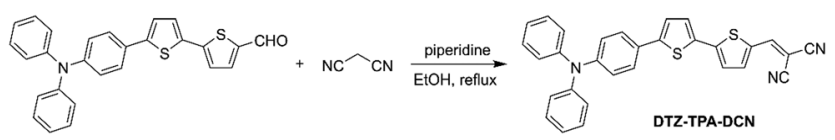

Scheme 1 The synthetic route and structure of DTZ-TPA-DCN.
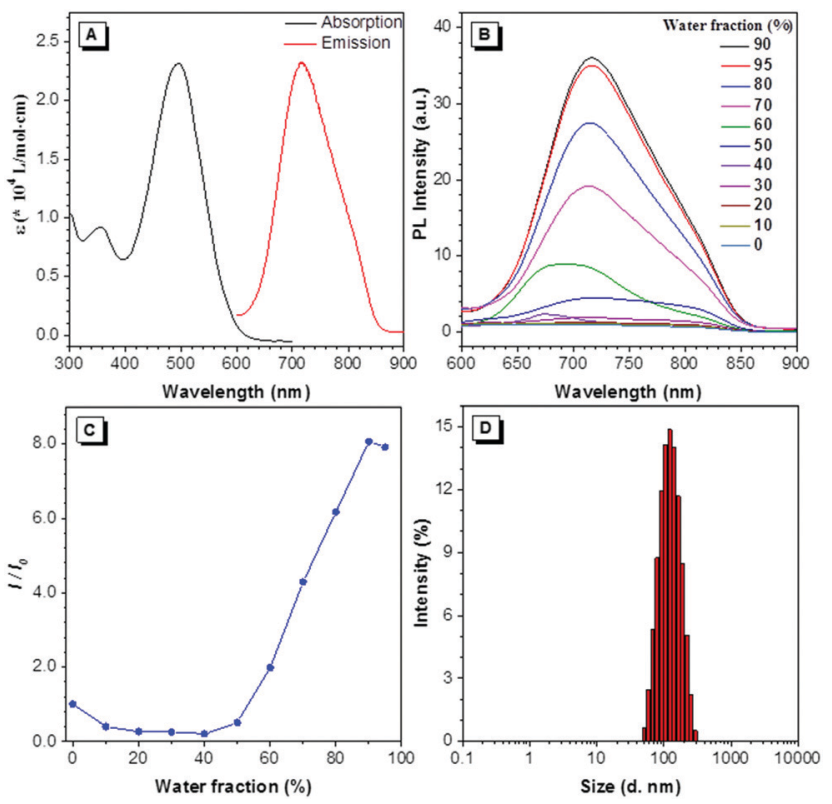

Fig. 1 General optical properties of DTZ-TPA-DCN. (A) Molar absorption and emission spectra of DTZ-TPA-DCN. (B) Photoluminescence (PL) intensity of DTZ-TPA-DCN $(10 \mu \mathrm{M})$ in mixtures of MeCN and water with different water contents. (C) Plots of the relative emission intensity of DTZ-TPA-DCN versus water fraction. $I_{0}$ and $/$ are the peak values of the PL intensities of DTZ-TPA-DCN $(10 \mu \mathrm{M})$ in $\mathrm{MeCN}$ and $\mathrm{MeCN} /$ water mixtures, respectively. (D) Size distribution of DTZ-TPA-DCN in the mixture of $\mathrm{MeCN} /$ water with $90 \%$ water content.

is $2.3 \%$, which is much higher than that observed in the aggregated and solution states. As a result, these data affirmatively revealed the AIE characteristics of DTZ-TPA-DCN.

\section{Cytotoxicity and co-localization experiment}

The cytotoxicity of DTZ-TPA-DCN in live cells was evaluated using a standard Cell Counting Kit-8 (CCK-8) assay, and HepG2 cells were treated with different concentrations of DTZ-TPADCN (from 2.5 to $20 \mu \mathrm{M}$ ). As illustrated in Fig. S2 (ESI $\dagger$ ), the cells were still highly viable even after incubated with $20 \mu \mathrm{M}$ DTZ-TPA-DCN for $24 \mathrm{~h}$, which demonstrated an excellent biocompatibility of DTZ-TPA-DCN. We further examined the cellular uptake and the subcellular pattern of DTZ-TPA-DCN in HepG2 cells. After incubating the cells with $10 \mu \mathrm{M}$ DTZ-TPADCN for $60 \mathrm{~min}$, a number of punctate structures in the cytoplasm were observed under the confocal laser scanning microscopy. These puncta highly colocalized with the commercial LD probe BODIPY 493/503 (Fig. 2A-C) with a Pearson's correlation coefficient $\left(R_{\mathrm{r}}\right)$ of 0.937 (Fig. S3, ESI $\dagger$ ), which indicated the high labelling specificity of DTZ-TPA-DCN to LDs. Besides live-cell imaging, DTZ-TPA-DCN also demonstrated a good performance in fixed cell imaging (Fig. S4, ESI $\dagger$ ). Moreover, as depicted in Fig. 2, 85\% of the fluorescence intensity of DTZ-TPA-DCN-stained LDs remained unchanged after 100 repetitive scanning under confocal microscopy, while the fluorescence intensity of BODIPY 493/503-stained LDs dropped to only $30 \%$ of its original value when using the same scanning parameter (Fig. 2D). Compared with BODIPY 493/503, 

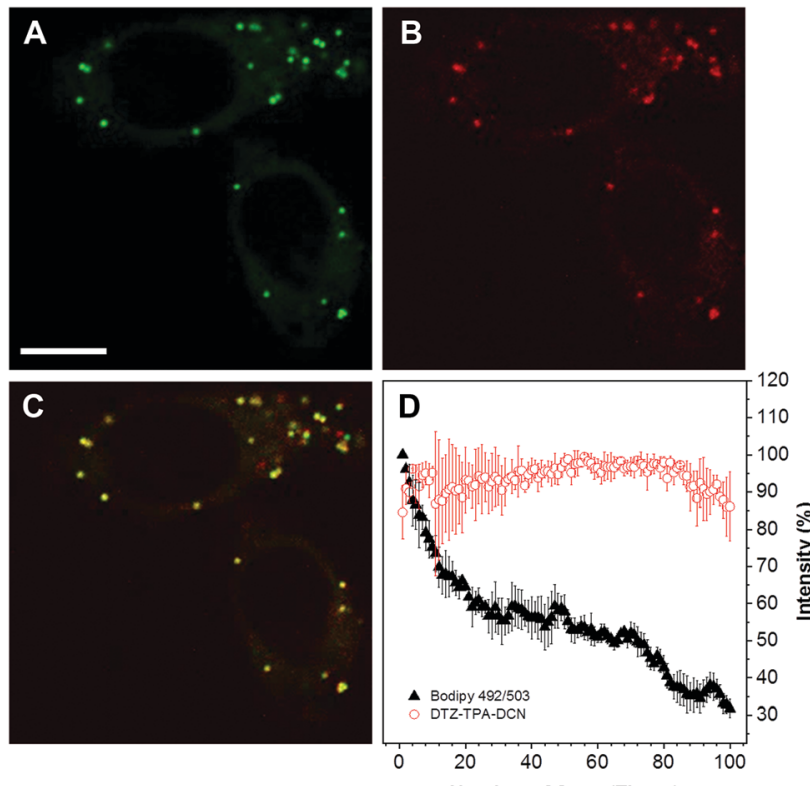

Fig. 2 Fluorescence imaging of HepG2 cells stained with BODIPY 493/503 and DTZ-TPA-DCN. (A and B) LDs were labelled by (A) BODIPY 493/503 and (B) DTZ-TPA-DCN. (C) Merged fluorescence and brightfield images. Scale bar: $10 \mu \mathrm{m}$. BODIPY: $493 / 503, \lambda_{\mathrm{ex}}=488 \mathrm{~nm}, \lambda_{\mathrm{em}}=510-540 \mathrm{~nm}$; DTZ-TPA-DCN: $\lambda_{\mathrm{ex}}=514 \mathrm{~nm}, \lambda_{\mathrm{em}}=550-650 \mathrm{~nm}$. (D) Fluorescence intensity of BODIPY 493/503 (solid triangle) and DTZ-TPA-DCN (open circle) after 100 times of scanning by a confocal laser scanning microscope.

DTZ-TPA-DCN showed a superior photostability, and this prompted further investigation of its performance under super-resolution fluorescence imaging, which could only be achieved using the fluorophores with an outstanding photostability. ${ }^{21}$

\section{Super-resolution imaging of LDs with SIM}

There have been few reports using AIE probes for SIM imaging till now. ${ }^{22}$ To test the feasibility of using DTZ-TPA-DCN for super-resolution imaging, SIM was used in the imaging of DTZ-TPA-DCN-stained LDs in HepG2 cells pre-treated with oleic acid (OA). OA is a fatty acid that exists naturally in numerous animal fats and vegetable oils, which can stimulate LD formation in both nucleus and cytoplasm by activating the longchain fatty acid receptor. ${ }^{23}$ After treating HepG2 cells with $0.4 \mathrm{mM}$ OA for $24 \mathrm{~h}$, not only the quantity but also the diameters of LDs in HepG2 cells were increased (Fig. S5, ESI $\dagger$ ). As shown in Fig. 3, the LD image acquired by the SIM exhibited an enhanced spatial resolution and an improved image quality compared with the images obtained from the confocal laser scanning microscopes (Nikon and ZEISS LSM 880, Fig. 3A, C and Fig. S6, ESI $\dagger$ ). With respect to SIM, LDs with sharper signals were observed, making them easier to be distinguished from each other in clusters, while the confocal images showed fuzzy LD signals. The line-scan plot of individual LD also demonstrated an enhanced spatial resolution (Fig. 3B and D), where the lateral full-width at half maximum (FWHM) of LDs in the SIM imaging was narrower than those observed in confocal
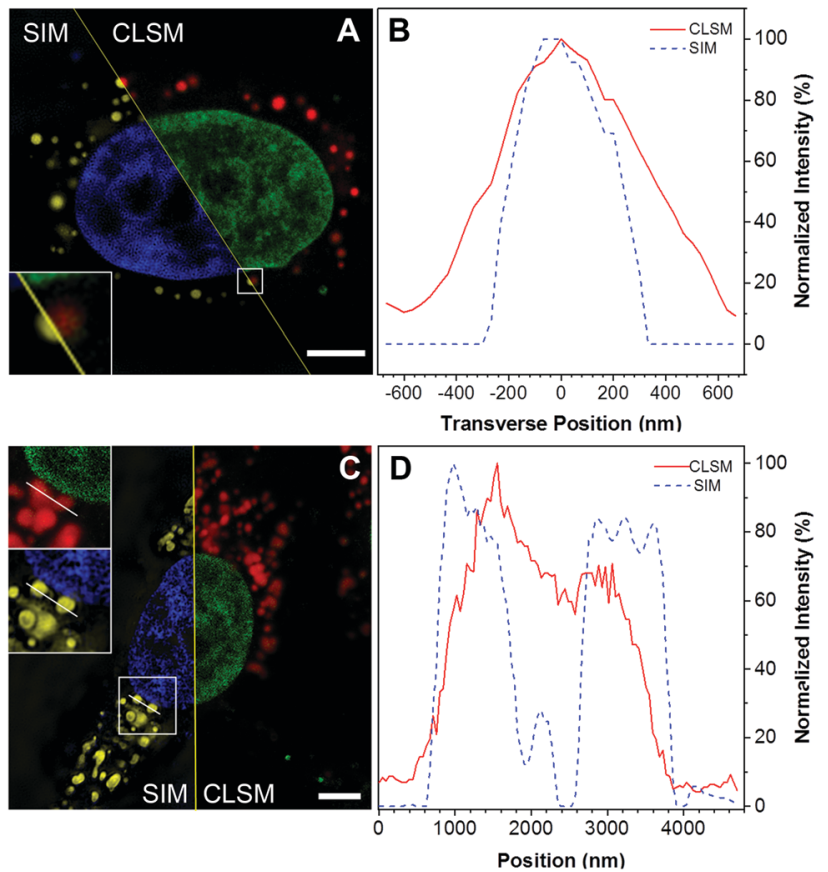

Fig. 3 DTZ-TPA-DCN-stained HepG2 cells captured by the structured illumination microscope (SIM) and confocal microscope. (A and C) The image on the left half is captured by the SIM, where the nucleus is highlighted in blue and the lipid droplets in yellow. The image on the right half is captured by the confocal microscope, where the nucleus is labelled in green and lipid droplets in red. Scale bar: $5 \mu \mathrm{m}$. (B) The super-resolution image of the lipid droplets showed more specific fluorescent signals with an FWHM of $450 \mathrm{~nm}$, which was narrower compared to the signals from the confocal microscope $(672 \mathrm{~nm})$. (D) Compared to the image obtained from CLSM, the super-resolution image showed a sharper signal that aided in the discrimination of the two adjacent lipid droplets from each other easily.

imaging (Fig. 3B). These results suggested that DTZ-TPA-DCN was applicable for imaging LDs by SIM.

\section{Fluorescence imaging of nLDs with DTZ-TPA-DCN}

Aside from the cytoplasmic LDs that originated from ER, the nLDs generated in the INM have drawn considerable attention because of its non-canonical role in the nuclear lipid homeostasis. ${ }^{14,15}$ However, the physiological functions, cellular regulatory machinery, and membrane contact site of nLDs remain to be explored. Taking advantage of DTZ-TPA-DCN possessing near-infrared emission, good LD selectivity, and practical application to super-resolution imaging, we applied this fluorophore to the study of nLDs. After treating HepG2 cells with OA, the cells were counterstained with $10 \mu \mathrm{M}$ DTZ-TPA-DCN and $20 \mu \mathrm{M}$ Hoechst 33342. As shown in Fig. 4A, most near-infrared DTZ-TPA-DCN puncta localized around the Hoechst-labelled nuclei. However, there were several DTZ-TPA-DCN-labelled LDs localized inside the nuclei, which were possibly the nLDs (Fig. 4A, white arrowheads). Through the $3 \mathrm{D}$ reconstructed images obtained by the Z-stack acquisition, we further verified that these red puncta were localized inside the nuclei instead of in close proximity to the nuclear surface (Fig. 4B and Fig. S7, Video S1, ESI $\dagger$ ). 

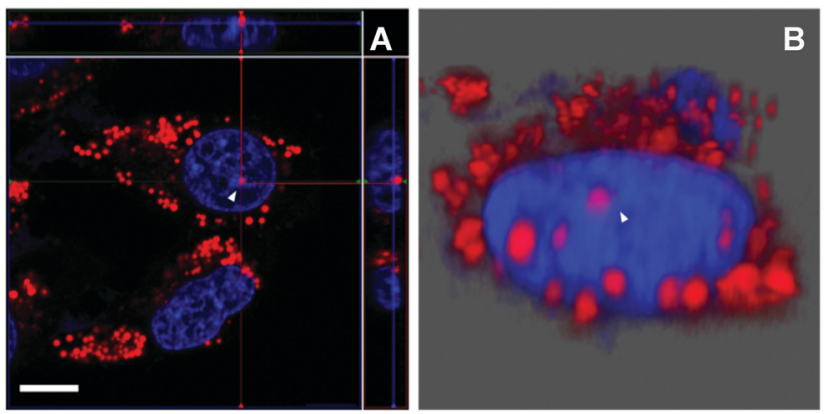

Fig. 4 Analysis of nLDs in HepG2 cells treated with OA. The fluorescence images were acquired by confocal laser scanning microscopy. The nuclei of the HepG2 cells were stained by Hoechst 33342 (blue), and the nLDs were stained by DTZ-TPA-DCN (red). (A) The image on the edge represents the orthogonal maximum intensity projection of the cells. (B) The rotated 3D reconstruction of the cell. The white arrowheads in both the images indicate the location of the $\mathrm{nLD}$. Scale bar: $5 \mu \mathrm{m}$.

As indicated by the white arrowheads in Fig. 4, the red signal is suspended in the cell nuclei. These results demonstrated the ability of DTZ-TPA-DCN to label nLDs and prompted us to further track the dynamics of nLDs by DTZ-TPA-DCN.

\section{Monitor the nLD dynamics}

To further examine the capability of DTZ-TPA-DCN in the monitoring of nLD dynamics, drugs which induced or suppressed the formation of nLDs were applied to the OA-treated HepG2 cells. It was reported that ER stress led to the formation of the nLDs, ${ }^{14,24}$ and tunicamycin (TM) induced the ER stress, which in turn enhanced the nLD formation in OA-treated hepatic cell lines. ${ }^{25}$ In our experiment, after treating the HepG2 cells with $0.4 \mathrm{mM}$ OA and $5 \mu \mathrm{gmL}^{-1} \mathrm{TM}$ for $12 \mathrm{~h}$, the cells were stained with DTZ-TPA-DCN, followed by imaging. We then counted and analyzed the percentage of cells enriched with nLDs in each population with different treatments. For the statistical analysis, around 100 cells were sampled for each of the population in triplicate. As shown in Fig. 5 and Fig. S8 (ESI $\dagger$ ), the OA-treated group shows a drastic increase in the nLDs compared with that of the control group. When TM was further added into the OA-treated group, the percentage of cells with nLDs also increased from $42.2 \%$ to $57.9 \%$ (Fig. 5A-C and E).
On the other hand, CP-346086, a microsomal triglyceride transfer protein (MTP) activity inhibitor, was used to suppress the nLD formation. ${ }^{26}$ When the OA/TM-treated cells were further treated with CP-346086, the percentage of cells with nLDs dropped and restored to a comparable level as compared to the group with the OA treatment only (Fig. 5D and E). Moreover, DTZ-TPA-DCN staining also revealed that CP-346086 could suppress the nLD formation with the OA treatment (Fig. S9 and S10, ESI $\dagger$ ). These results showed that DTZ-TPA-DCN could be used for the monitoring of the nLD formation. By using DTZ-TPA-DCN, the nLD dynamics in the hepatic cell lines was successfully tracked.

In addition, researches have shown that the active lipid metabolism and the distinct lipid composition of the INM affect the ability of the cells to generate nLDs. ${ }^{15}$ For instance, triacylglycerols (TAGs), which are the major neutral lipids stored in LDs, are essential factors in the biogenesis of LDs. ${ }^{1 b, 27}$ To promote the formation of LDs, the TAG in cells needs to reach a critical concentration. ${ }^{28}$ Moreover, the synthesis of TAGs from DAGs was catalysed by diacylglycerol acyltransferase (DGAT). Interestingly, DAGs were also highly related to the formation of nLDs. ${ }^{1 b, 27,29}$ Therefore, we would like to investigate whether the addition of DAG could promote the hepatocellular nLD formation. As shown in Fig. 6 and Fig. S11 (ESI $\dagger$ ), by using DTZ-TPA-DCN staining, we can observe that the nLDs were unevenly dispersed in cells with $0.4 \mathrm{mM}$ OA treatment. Interestingly, the percentage of cells containing nLDs increased from around $44.5 \%$ to more than $61.4 \%$ when the cells were treated with $0.4 \mathrm{mM}$ OA and DAG $(10 \mu \mathrm{M})$, suggesting that DAG enhanced the formation of nLDs in the hepatic cells (Fig. 6C). Some researchers pointed out that the seipin-deficient yeasts might form more nLDs when compared to the wild-type yeasts, and DAG is one of the factors involved in this phenomenon. The lack of seipin is believed to cause an increase in phosphatidic acid (PA), a precursor of DAG, in yeasts. When the free PA level inside the cells increased, excess PA would diffuse into the INM. These PAs would then be converted to DAG by PA phosphatases, which further upregulated the TAG synthesis and eventually contributed to more nLD formation. ${ }^{15,27}$ Despite this pathway was demonstrated using yeasts as a model organism only, it was postulated that the connection of lipid metabolism and nLD biogenesis were evolutionarily conserved in higher eukaryotes. This experiment suggested that DTZ-TPA-DCN could be a useful

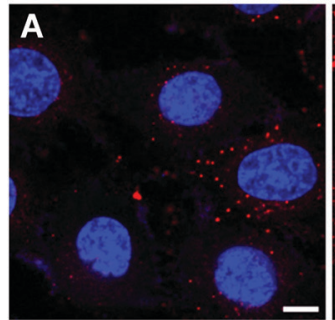

Control

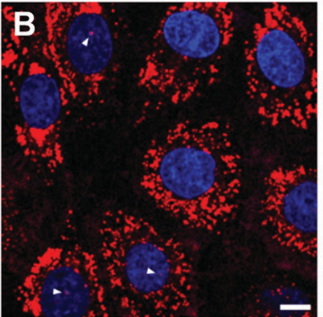

OA

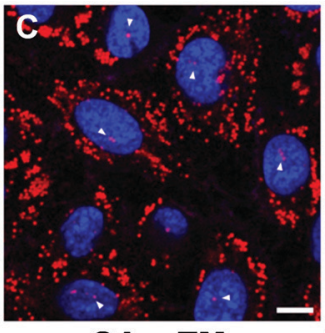

OA + TM

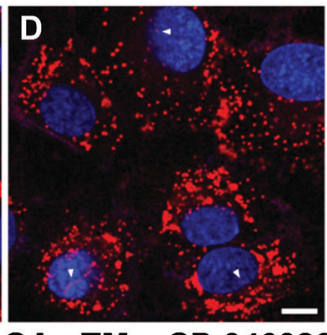

$\mathrm{OA}+\mathrm{TM}+\mathrm{CP}-346086$

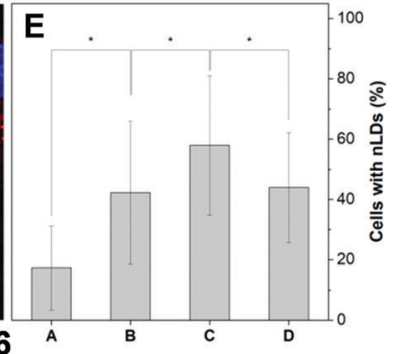

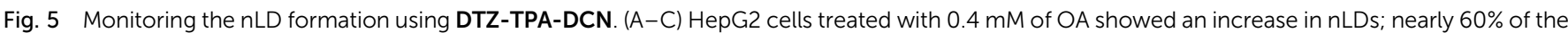

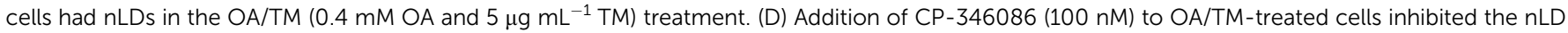

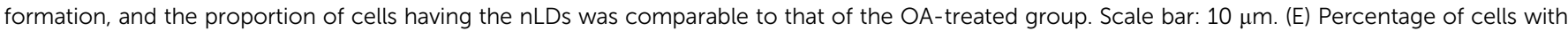

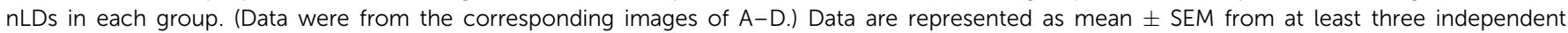
experiments. $n=29 / 30 / 25 / 27,{ }^{\star} P<0.05$. 

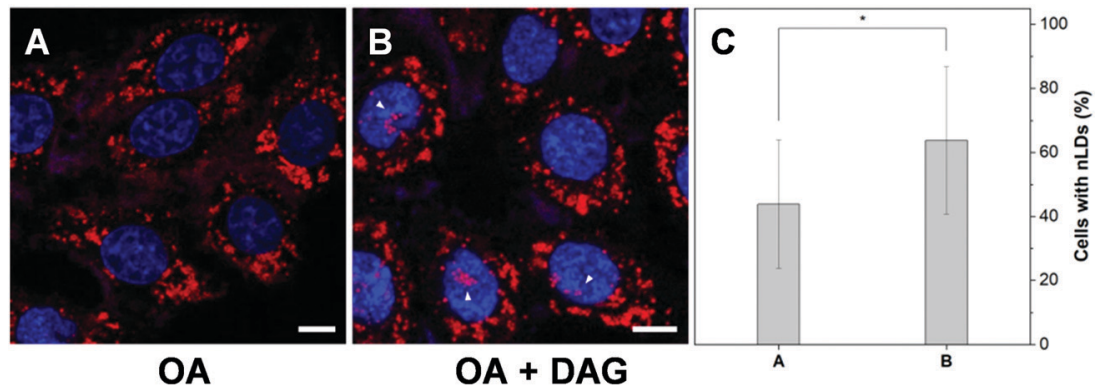

Fig. 6 Monitoring the $n L D$ change using DTZ-TPA-DCN during the DAG treatment. (A and B) Fluorescence images of the OA (0.4 mM) and OA (0.4 mM) plus DAG $(10 \mu \mathrm{M})$-treated group acquired by confocal laser scanning microscopy. Scale bar: $10 \mu \mathrm{m}$. (C) The percentage of the HepG2 cells with nLDs increased from around $44.5 \%$ to $61.4 \%$ when the cells were further treated with DAG. Data were from the corresponding images of (A) and (B). Data are represented as mean \pm SEM from at least three independent experiments. $n=31 / 29,{ }^{*} P<0.05$.

tool to explore the unravelled mechanism of nLD biogenesis and regulation.

\section{Conclusions}

In summary, a lipophilic near-infrared fluorescent probe, namely DTZ-TPA-DCN, was developed based on a common $\mathrm{D}-\pi-\mathrm{A}$ framework via a simple Knoevenagel condensation reaction. DTZ-TPA-DCN exhibited AIE properties with large Stokes shift and a distinct solvatochromic effect with high sensitivity towards environmental polarity. It was highly biocompatible and was able to label LDs specifically in both live and fixed cells. Owing to its excellent photostability, DTZ-TPADCN was successfully used in the SIM imaging of LDs. Furthermore, the nLDs were clearly observed in $2 \mathrm{D}$ or $3 \mathrm{D}$ imaging using DTZ-TPA-DCN in the labelling. The favourable optical properties of DTZ-TPA-DCN enabled further monitoring and tracking of the nLD dynamics under ER stress or by a lipid metabolism of the INM. It was shown for the first time that the important signaling lipid, DAG, induced nLD formation in mammalian cells. Conclusively, this study provided a versatile and reliable tool, DTZ-TPA-DCN, in the studies of nLDs, and its great potential for exploring the functional significance of nLDs was envisioned.

\section{Conflicts of interest}

There are no conflicts of interest to declare.

\section{Acknowledgements}

M.-Y. W. thanks the National Natural Science Foundation of China (21708030) and the Applied Basic Research of Sichuan Province (2021YJ0397). S. C. acknowledges the start-up funding from Ming Wai Lau Centre for Reparative Medicine, Karolinska Institutet.

\section{Notes and references}

1 (a) T. C. Walther and R. V. Farese Jr, Lipid droplets and cellular lipid metabolism, Annu. Rev. Biochem., 2012, 81,
687-714; (b) A. R. Thiam, R. V. Farese Jr and T. C. Walther, The biophysics and cell biology of lipid droplets, Nat. Rev. Mol. Cell Biol., 2013, 14, 775-786.

2 J. A. Olzmann, C. M. Richter and R. R. Kopito, Spatial regulation of UBXD8 and p97/VCP controls ATGLmediated lipid droplet turnover, Proc. Natl. Acad. Sci. U. S. A., 2013, 110, 1345-1350.

3 J. K. Zehmer, Y. Huang, G. Peng, J. Pu, R. G. W. Anderson and P. Liu, A role for lipid droplets in inter-membrane lipid traffic, Proteomics, 2009, 9, 914-921.

4 S. Martin and R. G. Parton, Lipid Droplets: A unified view of a dynamic organelle, Nat. Rev. Mol. Cell Biol., 2006, 7, 373-378.

5 (a) J. A. Olzmann and P. Carvalho, Dynamics and functions of lipid droplets, Nat. Rev. Mol. Cell Biol., 2019, 20, 137-155; (b) J. Boren and K. M. Brindle, Apoptosis-induced mitochondrial dysfunction causes cytoplasmic lipid droplet formation, Cell Death Differ., 2012, 19, 1561-1570.

6 (a) L. Tirinato, F. Pagliari, T. Limongi, M. Marini, A. Falqui, J. Seco, P. Candeloro, C. Liberale and E. Di Fabrizio, An overview of lipid droplets in cancer and cancer stem cells, Stem Cells Int., 2017, 2017, 1656053; (b) Q. Liu, Q. Luo, A. Halim and G. Song, Targeting lipid metabolism of cancer cells: a promising therapeutic strategy for cancer, Cancer Lett., 2017, 401, 39-45.

7 N. Krahmer, R. V. Farese and T. C. Walther, Balancing the fat: lipid droplets and human disease, EMBO Mol. Med., 2013, 5, 973-983.

8 G. Onal, O. Kutlu, D. Gozuacik and S. D. Emre, Lipid droplets in health and disease, Lipids Health Dis., 2017, 16, 128.

9 A. Paul, B. H.-J. Chang, L. Li, V. K. Yechoor and L. Chan, Deficiency of adipose differentiation-related protein impairs foam cell formation and protects against atherosclerosis, Circ. Res., 2008, 102, 1492-1501.

10 E. Kiss, B. Kranzlin, K. Wagenbala $\beta$, M. Bonrouhi, J. Thiery, E. Grone, V. Nordstrom, D. Teupser, N. Gretz, E. Malle and H.-J. Grone, Lipid droplet accumulation is associated with an increase in hyperglycemia-induced renal damage: prevention by liver X receptors, Am. J. Pathol., 2013, 182, 727-741. 
11 N. Roitenberg and E. Cohen, Lipid assemblies at the crossroads of aging, proteostasis, and neurodegeneration, Trends Cell Biol., 2019, 29, 954-963.

12 (a) V. Choudhary, G. Golani, A. S. Joshi, S. Cottier, R. Schneiter, W. A. Prinz and M. M. Kozlov, Architecture of lipid droplets in endoplasmic reticulum is determined by phospholipid intrinsic curature, Curr. Biol., 2018, 19(28), 915-926; (b) T. C. Walther and R. V. Farese Jr, Lipid droplets and cellular lipid metabolism, Annu. Rev. Biochem., 2012, 81, 687-714.

13 (a) J. P. Layerenza, P. González, M. M. García de Bravo, M. P. Polo, M. S. Sisti and A. Ves-Losada, Nuclear lipid droplets: a novel nuclear domain, Biochim. Biophys. Acta, 2013, 1831, 327-340; (b) R. Uzbekov and P. Roingeard, Nuclear lipid droplets identified by electron microscopy of serial sections, BMC Res. Notes, 2013, 6, 386; (c) L. Wang, Y. Wang, Y. Liang, J. Li, Y. Liu, J. Zhang, A. Zhang, J. Fu and G. Jiang, Physical key-protected one-time pad, Sci. Rep., 2013, 3, 217.

14 K. Soltysik, Y. Ohsaki, T. Tatematsu, J. Cheng and T. Fujimoto, Nuclear lipid droplets derive from a lipoprotein precursor and regulate phosphatidylcholine synthesis, Nat. Commun., 2019, 10, 473.

15 A. Romanauska and A. Köhler, The inner nuclear membrane is a metabolically active territory that generates nuclear lipid droplets, Cell, 2018, 174, 700-715.

16 (a) R. Yuste, Fluorescence microscopy today, Nat. Methods, 2005, 2, 902-904; (b) J. W. Lichtman and J.-A. Conchello, Fluorescence microscopy, Nat. Methods, 2005, 2, 910-919.

17 (a) A. Malhas, C. Goulbourne and D. J. Vaux, The nucleoplasmic reticulum: form and function, Trends Cell Biol., 2011, 21, 362-373; (b) R. Bernardi and P. P. Pandolfi, Structure, dynamics and functions of promyelocytic leukaemia nuclear bodies, Nat. Rev. Mol. Cell Biol., 2007, 8, 1006-1016; (c) Y. Ohsaki, T. Kawai, Y. Yoshikawa, J. Cheng, E. Jokitalo and T. Fujimoto, PML isoform II plays a critical role in nuclear lipid droplet formation, J. Cell Biol., 2016, 212, 29-38.

18 (a) M. Collot, T. K. Fam, P. Ashokkumar, O. Faklaris, T. Galli, L. Danglot and A. S. Klymchenko, Ultrabright and fluorogenic probes for multicolor imaging and tracking of lipid droplets in cells and tissues, J. Am. Chem. Soc., 2018, 140, 5401-5411; (b) H. Xu, H. Zhang, G. Liu, L. Kong, X. Zhu, X. Tian, Z. Zhang, R. Zhang, Z. Wu, Y. Tian and H. Zhou, Coumarin-based fluorescent probes for super-resolution and dynamic tracking of lipid droplets, Anal. Chem., 2019, 91, 977-982; (c) M. Collot, S. Bou, T. K. Fam, L. Richert, Y. Mely, L. Danglot and A. S. Klymchenko, Probing polarity and heterogeneity of lipid droplets in live cells using a pushpull fluorophore, Anal. Chem., 2019, 91, 1928-1935; (d) D. Dang, H. Liu, J. Wang, M. Chen, Y. Liu, H. H.-Y. Sung, I. D. Williams, R. T. K. Kwok, J. W. Y. Lam and B. Z. Tang, Highly emissive AIEgens with multiple functions: facile synthesis, chromism, specific lipid droplet imaging, apoptosis monitoring, and in vivo imaging, Chem. Mater., 2018, 30, 7892-7901; (e) T. K. Fam,
A. S. Klymechenko and M. Collot, Recent advances in fluorescent probes for lipid droplets, Materials, 2018, 11, 1768; $(f)$ D. Wang, H. Su, R. T. K. Kowk, G. Shan, A. C. S. Leung, M. M. S. Lee, H. H. Y. Sung, I. D. Williams, J. W. Y. Lam and B. Z. Tang, Facile synthesis of red/NIR AIE luminogens with simple structures, bright emissions, and high photostabilities, and their applications for specific imaging of lipid droplets and image-guided photodynamic therapy, Adv. Funct. Mater., 2017, 27, 1704039; $(g)$ L. Guo, M. Tian, R. Feng, G. Zhang, R. Zhang, X. Li, Z. Liu, X. He, J. Z. Sun and X. Yu, Interface-targeting strategy enables twophoton fluorescent lipid droplet probes for high-fidelity imaging of turbid tissues and detecting fatty liver, ACS Appl. Mater. Interfaces, 2018, 10, 10706-10717; (h) L. Shi, K. Li, L.-L. Li, S.-Y. Chen, M.-Y. Li, Q. Zhou, N. Wang and X.-Q. Yu, Novel easily available purine-based AIEgens with colour tunability and applications in lipid droplet imaging, Chem. Sci., 2018, 9, 8969-8974; (i) J.-S. Ni, H. Liu, J. Liu, M. Jiang, Z. Zhao, Y. Chen, R. T. K. Kwok, J. W. Y. Lam, Q. Peng and B. Z. Tang, The unusual aggregation-induced emission of coplanar organoboron isomers and their lipid dropletspecific applications, Mater. Chem. Front., 2018, 2, 1498-1507; (j) N. Zhao, Y. Li, W. Yang, J. Zhuang, Y. Li and N. Li, Multifunctional pyrazoline based AIEgens: real-time tracking and specific protein "fishing" of lipid droplets, Chem. Sci., 2019, 10, 9009-9016; (k) W. Xu, M. M. S. Lee, Z. Zhang, H. H. Y. Sung, I. D. Willams, R. T. K. Kwok, J. W. Y. Lam, D. Wang and B. Z. Wang, Facile synthesis of AIEgens with wide color tunability for cellular imaging and therapy, Chem. Sci., 2019, 10, 3494-3501; (l) H. Tian Jr, A. C. Sedgwick, H.-H. Han, S. Sen, G.-R. Chen, Y. Zang, J. L. Sessler, T. D. James, J. Li and X.-P. He, Fluorescent probes for the imaging of lipid droplets in live cells, Coord. Chem. Rev., 2021, 427, 213577.

19 (a) J. Luo, Z. Xie, J. W. Y. Lam, L. Cheng, H. Chen, C. Qiu, H. S. Kowk, X. Zhan, Y. Liu, D. Zhu and B. Z. Tang, Aggregation-induced emission of 1-methyl-1,2,3,4,5-pentaphenylsilole, Chem. Commun., 2001, 1740-1741; (b) J. Mei, N. L. C. Leung, R. T. K. Kwok, J. W. Y. Lam and B. Z. Tang, Aggregation-induced emission: together we shine, united we soar!, Chem. Rev., 2015, 115(21), 11718-11940; (c) Y. Hong, J. W. Y. Lam and B. Z. Tang, Aggregationinduced emission, Chem. Soc. Rev., 2011, 40, 5361-5388; (d) X. Cai and B. Liu, Aggregation-induced emission: recent advances in materials and biomedical applications, Angew. Chem., Int. Ed., 2020, 59, 9868-9886; (e) S. Xu, Y. Duan and B. Liu, Precise molecular design for high-performance luminogens with aggregation-induced emission, $A d v$. Mater., 2019, 32, 1903530; $(f)$ Z. Guo, C. Yan and W.-H. Zhu, High-performance quinoline-malononitrile core as a building block for the diversity-oriented synthesis of AIEgens, Angew. Chem., Int. Ed., 2020, 59, 9812-9825; (g) J. Mei, Y. Huang and H. Tian, Progress and trends in AIE-based bioprobes: a brief overview, ACS Appl. Mater. Interfaces, 2018, 10, 12217-12261. 
20 (a) M.-Y. Wu, J.-K. Leung, L. Liu, C. Kam, K. Y. K. Chan, R. A. Li, S. Feng and S. Chen, A small-molecule AIE chromosome periphery probe for cytogenetic studies, Angew. Chem., Int. Ed., 2020, 59, 10327-10331; (b) L. Shi, Y.-H. Liu, K. Li, A. Sharam, K.-K. Yu, M. S. Ji, L.-L. Li, Q. Zhou, H. Zhang, J. S. Kim and X.-Q. Yu, An AIE-based probe for rapid and ultrasensitive imaging of plasma membranes in biosystems, Angew. Chem., Int. Ed., 2020, 59, 9962-9966.

21 L. Wang, M. S. Frei, A. Salim and K. Johnsson, Smallmolecule fluorescent probes for live-cell super-resolution microscopy, J. Am. Chem. Soc., 2019, 141, 2770-2781.

22 X. Ge, M. Gao, B. Situ, W. Feng, B. He, X. He, S. Li, Z. Ou, Y. Zhong, Y. Lin, X. Ye, X. Hu, B. Z. Tang and L. Zheng, Onestep, rapid fluorescence sensing of fungal viability based on a bioprobe with aggregation-induced emission characteristics, Mater. Chem. Front., 2020, 4, 957-964.

23 A. Rohwedder, Q. Zhang, S. A. Rudge and M. J. O. Wakelam, Lipid droplet formation in response to oleic acid in Huh-7 cells is mediated by the fatty acid receptor FFAR4, J. Cell Sci., 2014, 127, 3104-3115.
24 L. Ozcan and I. Tabas, Role of endoplasmic reticulum stress in metabolic disease and other disorders, Annu. Rev. Med., 2012, 63, 317-328.

25 J.-S. Lee, R. Mendez, H. H. Heng, Z.-Q. Yang and K. Zhang, Pharmacological ER stress promotes hepatic lipogenesis and lipid droplet formation, Am. J. Transl. Res., 2012, 4, 102-113.

26 G. F. Gibbons, K. Islam and R. J. Pease, Mobilisation of triacylglycerol stores, Biochim. Biophys. Acta, 2000, 1483, 37-57.

27 H. F. Hashemi and J. M. Goodman, The life cycle of lipid droplets, Curr. Opin. Cell Biol., 2015, 33, 119-124.

28 A. R. Thiam and M. Beller, The why, when and how of lipid droplet diversity, J. Cell Sci., 2017, 130, 315-324.

29 (a) A. D. Barbosa, K. Lim, M. Mari, J. R. Edgar, L. Gal, P. Sterk, B. J. Jenkins, A. Koulman, D. B. Savage, M. Schuldiner, F. Reggirori, P. A. Wigge and S. Siniossoglou, Compartmentalized synthesis of triacylglycerol at the inner nuclear membrane regulates nuclear organization, Dev. Cell, 2019, 50, 755-766; (b) M. Gao, X. Huang, L. Bao and H. Yang, The biogenesis of lipid droplets: Lipids take center stage, Prog. Lipid Res., 2019, 75, 100989. 